\title{
Permanent-Magnet Helicon Discharge Array
}

\author{
Francis F. Chen and Humberto Torreblanca
}

\begin{abstract}
Industrial applications of plasmas often require uniform coverage of large areas, such as in plasma coatings, display panels, and semiconductor wafers. A single source would have to be very large, but an array of small sources can be much more compact and yet cover an arbitrarily large area. Such sources can be inductively coupled plasmas (ICPs) which are powered by radio-frequency generators. Helicon-wave sources have been found to create higher plasma densities than ICPs at the same power, but these require a dc magnetic field, which normally would entail large and heavy electromagnets and their dc power supplies. Annular permanent magnets can produce helicon plasmas if used in the innovative way described here. An eight-tube array of such sources has been built and tested.
\end{abstract}

Index Terms-Helicon discharges, large-area plasmas, plasma applications, plasma sources.

$\mathbf{F}$ OR PROCESSING large substrates with high-density lowpressure plasmas, an array of small sources can produce uniform coverage within a few percent. Helicon sources are known for their high ionization efficiency, but they require a dc magnetic field, which normally would be an electromagnet large enough to cover the entire area. Here, the B-field is produced by small annular permanent magnets, one for each tube, lying in a tray above the discharges. Fig. 3 shows the photograph of the Medusa 2 machine, which consists of an array of eight tubes driven in parallel by a 3.2-kW RF supply at 13.56 MHz. Between the two staggered rows of tubes is a $50-\Omega$ rectangular transmission line in which the center conductor is a 1/4-in-diameter (6.4-mm-diameter) copper pipe which carries both the RF current and the cooling water. The three-turn $m=0$ antennas are of 1/8-in-diameter (3.2-mm-diameter) copper tubing and are cooled in pairs so that the water enters and leaves at ground potential. The quartz discharge tubes are 2 in $(5 \mathrm{~cm})$ in diameter and are 2 in high, and the system operates in the steady state. The tube axes are 7 in $(17.8 \mathrm{~cm})$ apart, as are the row center lines. The tubes in each row can be opposite from one another or staggered, as is shown in the picture. The argon gas is normally at $20 \mathrm{mtorr}$, but pressures between 1 and 200 mtorr are possible.

The use of the reverse external field of the ring magnets allows the plasma to follow the slowly diverging field lines downward to the substrate. The behavior of the B-field can be

Manuscript received November 8, 2010; revised May 6, 2011, May 10, 2011, and June 9, 2011; accepted June 16, 2011. Date of publication August 4, 2011; date of current version November 9, 2011.

The authors are with the Electrical Engineering Department, University of California, Los Angeles, CA 90095 USA (e-mail: ffchen@ee.ucla.edu; humberto.torreblanca@intel.com).

Color versions of one or more of the figures in this paper are available online at http://ieeexplore.ieee.org.

Digital Object Identifier 10.1109/TPS.2011.2160345

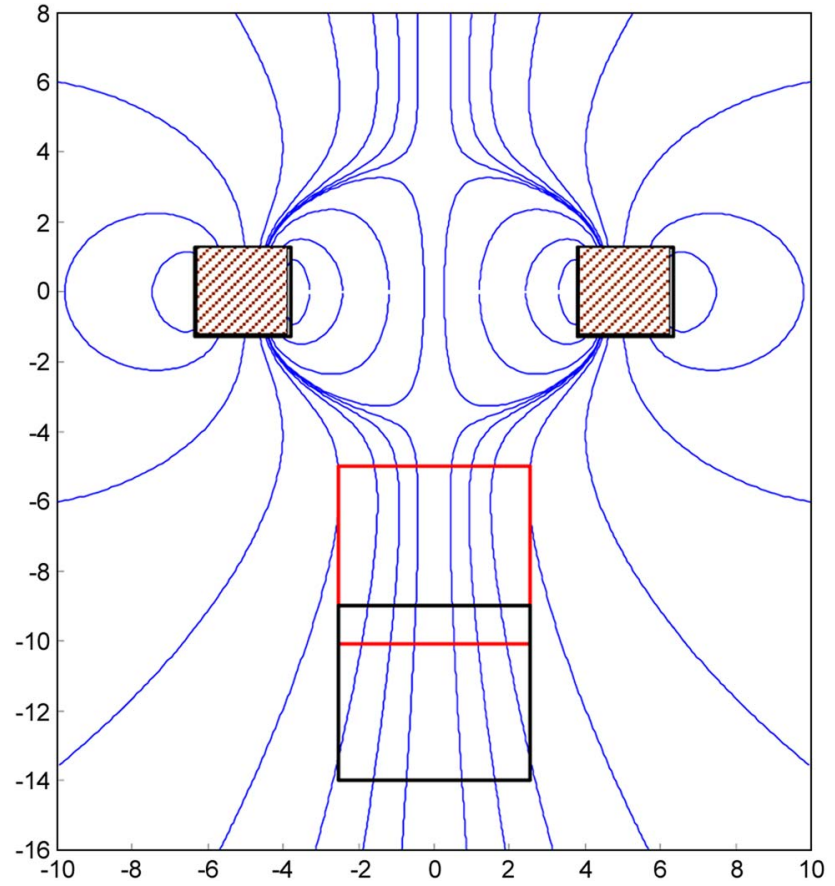

Fig. 1. Field lines of a NdFeB ring magnet of 3-in ID and 5-in OD with rectangles showing two possible positions for the discharge tube.

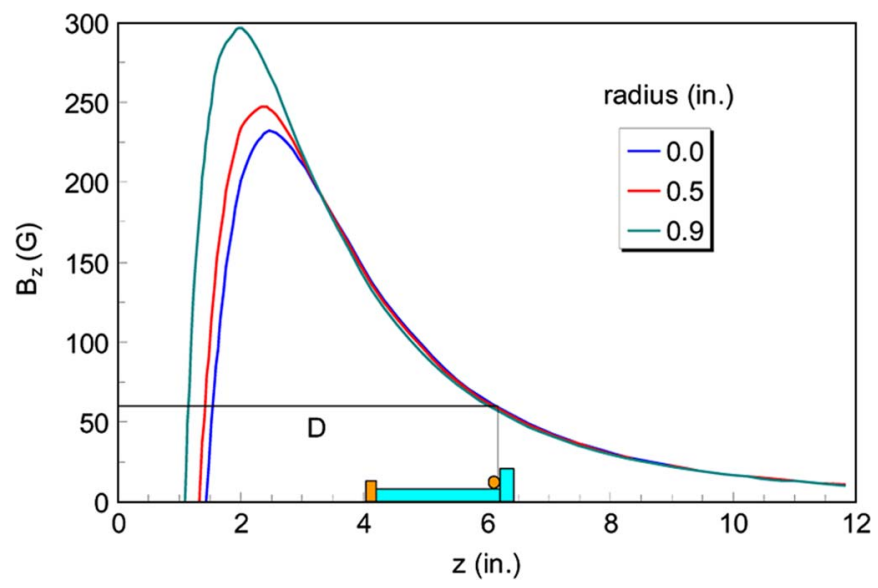

Fig. 2. Magnetic field versus distance from the magnet's midplane at three radial positions from its axis.

seen from the plasma shape. Since the field at the antenna is less than $100 \mathrm{G}$, the tube height is set by the condition that the helicon wave reflected from the aluminum top plate interferes constructively with the downward wave, enhancing the density at low fields. ${ }^{1}$ In this configuration, a plasma density of order $10^{11} \mathrm{~cm}^{-3}$, uniform to about $\pm 3 \%$, can be obtained on a plane

${ }^{1}$ Detailed calculations of this resonance condition were made with the HELIC code used in the design of helicon discharges. 


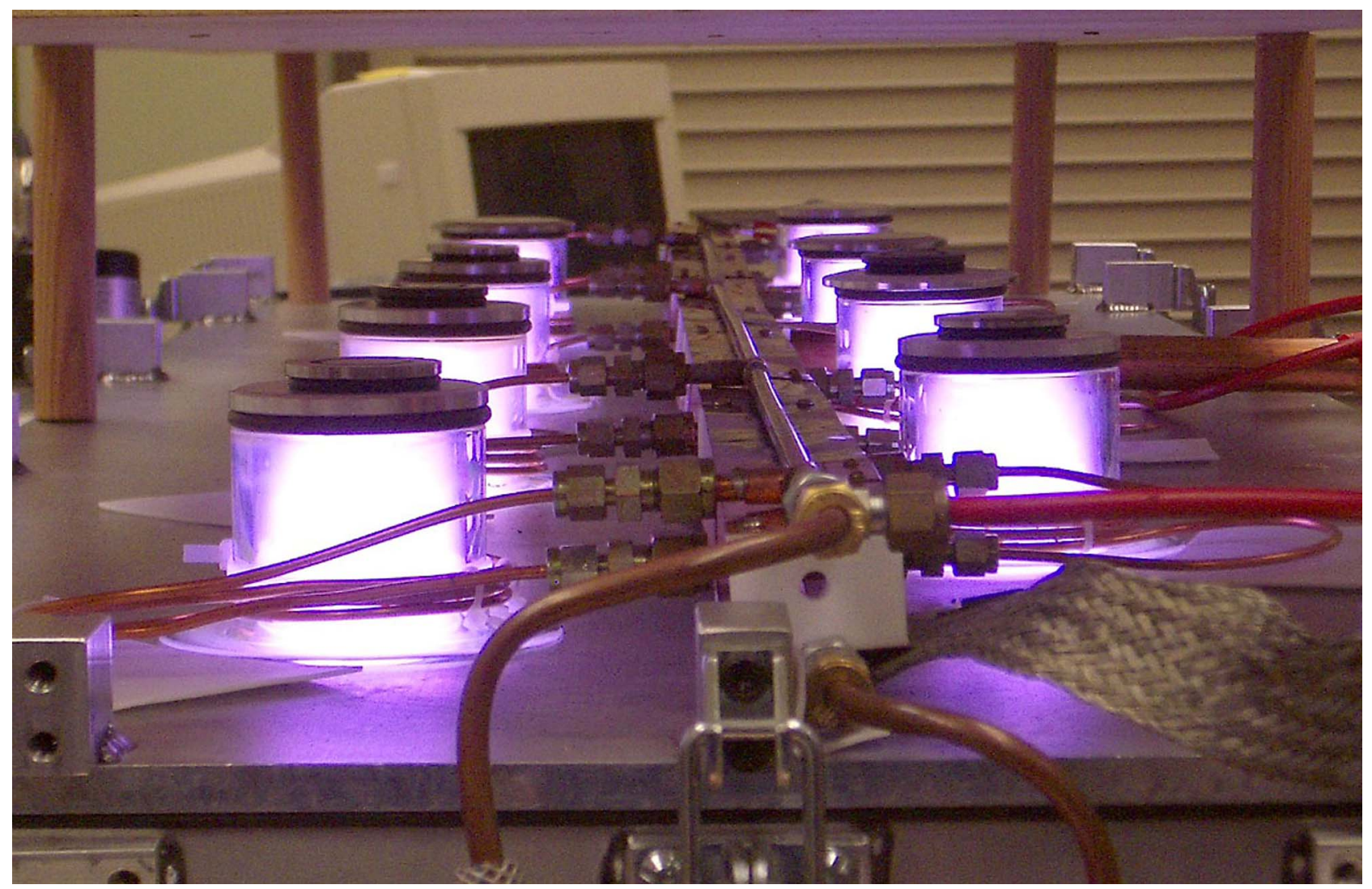

Fig. 3. Eight-tube array of helicon sources using annular permanent magnets located above them.

$z=19 \mathrm{~cm}$ below the source, where the field is $\leq 10 \mathrm{G}$. The uniformity is measured with a line of 15 probes across the array, movable in the $x$ direction (into the picture); its $z$ position was not varied in the time available. The array of tubes and magnets occupies only about 6 in $(16 \mathrm{~cm})$ of vertical space.

All eight tubes were driven in parallel from a single manual matching circuit. Getting all tubes to ignite equally is nontrivial and requires experimental skill. With insufficient power, not all tubes will ignite. Depending on the pressure, 300-400 W/tube is necessary to bring all the tubes from the ICP mode into the helicon mode. With the transmission line shown earlier, it is difficult to get equal power to the tubes farthest from the central feed point. It is better to use a central distribution point equally distant from each tube. However, geometric variations of this array built at other laboratories have shown nonuniformities which are not yet understood.

In designing the individual sources, the HELIC code [1] was used to optimize the tube diameter and height for highest plasma loading. Small tubes were used to achieve plasma uniformity not far below the tubes. Annular magnets were designed to give a fairly uniform B-field in the remote region, as shown in Fig. 1. Using the field below the stagnation point permits ejection of the plasma to the substrate. The B-field can be varied by moving the tube or magnet up or down, as shown by the two rectangles in Fig. 1. The computed field strength, calibrated with a gaussmeter, at various heights and radii is shown in Fig. 2. The much stronger field inside the magnet ring $\left(B_{z}<0\right)$ is not shown. The distance $D$ determines the field at the antenna. The final design strikes a sweet spot such that even the antenna inductance is suitable for matching at $13.56 \mathrm{MHz}$. Further details on this invention are given in previous publications [2]-[4].

\section{REFERENCES}

[1] D. Arnush, "The role of Trivelpiece-Gould waves in antenna coupling to helicon waves," Phys. Plasmas, vol. 7, no. 7, pp. 3042-3050, Jul. 2000.

[2] H. Torreblanca, "Multitube helicon source with permanent magnets," Ph.D. dissertation, UCLA, Los Angeles, CA, 2008.

[3] F. F. Chen and H. Torreblanca, "Large-area helicon plasma source with permanent magnets," Plasma Phys. Control. Fusion, vol. 49, no. 5A, pp. A81A93, May 2007.

[4] F. F. Chen and H. Torreblanca, "Permanent magnet helicon sources and arrays: A new type of RF plasma," Phys. Plasmas, vol. 16, no. 5, pp. 057 102-1-057 102-8, May 2009. 